\title{
Thymoma and Synchronous Primary Mediastinal Seminomas with Florid Follicular Lymphoid Hyperplasia in the Anterior Mediastinum: A Case Report and Review of the Literature
}

\author{
Hyang-im Lee ${ }^{1} \cdot$ In-seok Jang ${ }^{2,3,4}$ \\ Kyung Nyeo Jeon ${ }^{3,4,5}$ \\ Gyung Hyuck Ko ${ }^{3,4,6} \cdot$ Jong Sil Lee ${ }^{3,4,6}$ \\ Dong Chul Kim ${ }^{3,4,6} \cdot$ Dae Hyun Song ${ }^{1,3}$ \\ Jeong-Hee Lee 3,4, $^{3,6}$ \\ 'Department of Pathology, Gyeongsang National \\ University Changwon Hospital, Changwon; \\ ${ }^{2}$ Department of Thoracic and Cardiovascular \\ Surgery, Gyeongsang National University \\ Hospital, Jinju; ${ }^{3}$ Gyeongsang National University \\ School of Medicine, Jinju; ${ }^{4}$ nstitute of Health \\ Science, Gyeongsang National University, Jinju; \\ ${ }^{5}$ Department of Radiology, Gyeongsang National \\ University Changwon Hospital, Changwon; \\ ${ }^{6}$ Department of Pathology, Gyeongsang National \\ University Hospital, Jinju, Korea \\ Received: May 11, 2016 \\ Revised: August 11, 2016 \\ Accepted: August 24, 2016 \\ Corresponding Author \\ Jeong-Hee Lee, MD, PhD \\ Department of Pathology, Gyeongsang National \\ University School of Medicine, 15 Jinju-daero \\ 816beon-gil, Jinju 52727, Korea \\ Tel: +82-55-772-8062 \\ Fax: +82-55-759-7952 \\ E-mail: jhlee7@gnu.ac.kr
}

Thymoma is the most common neoplasm of the anterior mediastinum and has malignant potential. Germ cell tumors (GCTs) found in the anterior mediastinum are usually benign, and malignant GCTs, such as seminomas, are rare. Histologically, mediastinal seminoma is indistinguishable from testicular seminoma except for site-associated morphological features such as lymphoid follicular hyperplasia. Therefore, excluding metastasis is very important. Recently, we treated a young adult patient with multiple thymic masses that occurred simultaneously. The patient underwent a thymectomy for the removal of the mediastinal masses, one of which was diagnosed as type B2 invasive thymoma, and two of which were diagnosed as primary mediastinal seminomas with massive follicular hyperplasia. The patient received adjuvant chemotherapy after surgical resection. To our knowledge, this is the first description of a thymoma and a mediastinal seminoma occurring simultaneously in the thymus. We present this case along with a literature review.

Key Words: Thymoma; Seminoma; Mediastinum; Follicular hyperplasia
In general, thymomas are rare tumors; however, they are the most common mediastinal tumors in adults, comprising approximately $50 \%$ of all anterior mediastinal tumors. Germ cell tumors (GCTs) of the mediastinum are also uncommon in adults and account for $10 \%$ to $20 \%$ of anterior mediastinal tumors. ${ }^{1-3}$ Teratomas are the most common mediastinal GCTs, accounting for $44 \%$ of all mediastinal GCTs, and seminomas are the second most common, with an incidence of approximately $37 \%{ }^{4}$ Thymomas occur most frequently in adults between the ages of 55 and 65 years, and thus, they are exceedingly rare in children and adolescents. Seminomas are primarily seen in young adult patients. According to previous reports, thymomas are commonly associated with thymic epithelial tumors, and seminomas are occasionally associated with smooth muscle tumors or multilobular thymic cysts. ${ }^{5-7}$ Recently, Weissferdt et al. ${ }^{8}$ described two cases of adult men, who were 32 and 34 years old, with mediastinal tumors that contained both a thymoma and seminoma component in a single mass. These are very rare cases that have not been previously reported. Interestingly, our group examined a patient with multiple thymic masses including one thymoma and two seminomas occurring as separate tumors. The seminomas showed prominent lymphoid follicular hyperplasia. 
Because mediastinal seminomas are both rare and morphologically similar to their gonadal counterparts, the possibility of metastasis from testicular or ovarian tumors should always be excluded during the workup of these patients. Fortunately, mediastinal GCTs can be associated with secondary changes that are rarely found in testicular or ovarian tumors. These changes include reactive proliferation of remnant thymic epithelium, multiple cystic formations, epithelioid granulomatous reactions, fibrosis, and follicular lymphoid hyperplasia. ${ }^{4,79-11}$ To the best of our knowledge, the simultaneous occurrence of thymomas and seminomas in the thymus has not been previously reported. Here, we report a case with a brief review of the literature.

\section{CASE REPORT}

A 35-year-old man presented with a $7.0 \mathrm{~cm}$ anterior mediastinal mass on a chest radiograph during a routine medical evaluation at an outside hospital. The patient was transferred to the Department of Thoracic and Cardiovascular Surgery for further evaluation and treatment. The patient did not present with any specific medical problems or symptoms and had no family history of cancer or prior surgeries except for an operation due to a pneumothorax that was performed 12 years prior to presentation. A whole body radiological study was performed. Fluorodeoxyglucose (FDG)-positron emission tomography/computed tomography (CT) revealed two heterogenic hypermetabolic foci in the anterior mediastinum, with standard uptake values of 3.3 and 3.4. No significant FDG uptake was observed in the lymph nodes or anywhere else in the body to suggest distant metastasis, except for physiological uptake in both testes (Fig. 1A). Contrast-enhanced chest $\mathrm{CT}$ images showed multiple tumors in the anterior mediastinum. Specifically, a $5.0 \mathrm{~cm}$ mass compressing the superior vena cava, a $1.5 \mathrm{~cm}$ nodule, and a $1.0 \mathrm{~cm}$ nodule were observed (Fig. 1B, C). Radiologically, the differential diagnosis included invasive thymoma and GCT. Ultrasonography of the testes revealed a normal shape and echogenicity without focal mass or infiltrative lesions. The serum levels of $\beta$ human chorionic gonadotropin $(0.1 \mathrm{IU} / \mathrm{L})$ and $\alpha$-fetoprotein $(12.8 \mathrm{ng} / \mathrm{mL})$ were in the normal range.

A biopsy was performed in the operating room, and the diagnosis of thymoma was determined based on frozen tissue sections. The patient underwent a thymectomy to remove the mediastinal masses. The thymectomy specimen showed three well-circumscribed solid masses on cross section. The largest mass $(5.0 \times 5.0$ $\mathrm{cm}$ in size) showed a lobulated, homogeneous, firm, cut surface with a partial hemorrhage. In the normal thymic tissue surrounding the largest tumor, two smaller masses $(1.5 \times 1.2 \mathrm{~cm}$ and $1.0 \times 0.5 \mathrm{~cm}$ in size) were seen, which had a well-defined, round, homogeneous, soft appearance. The medium-sized mass was located $1.5 \mathrm{~cm}$ from the largest tumor, and the smallest
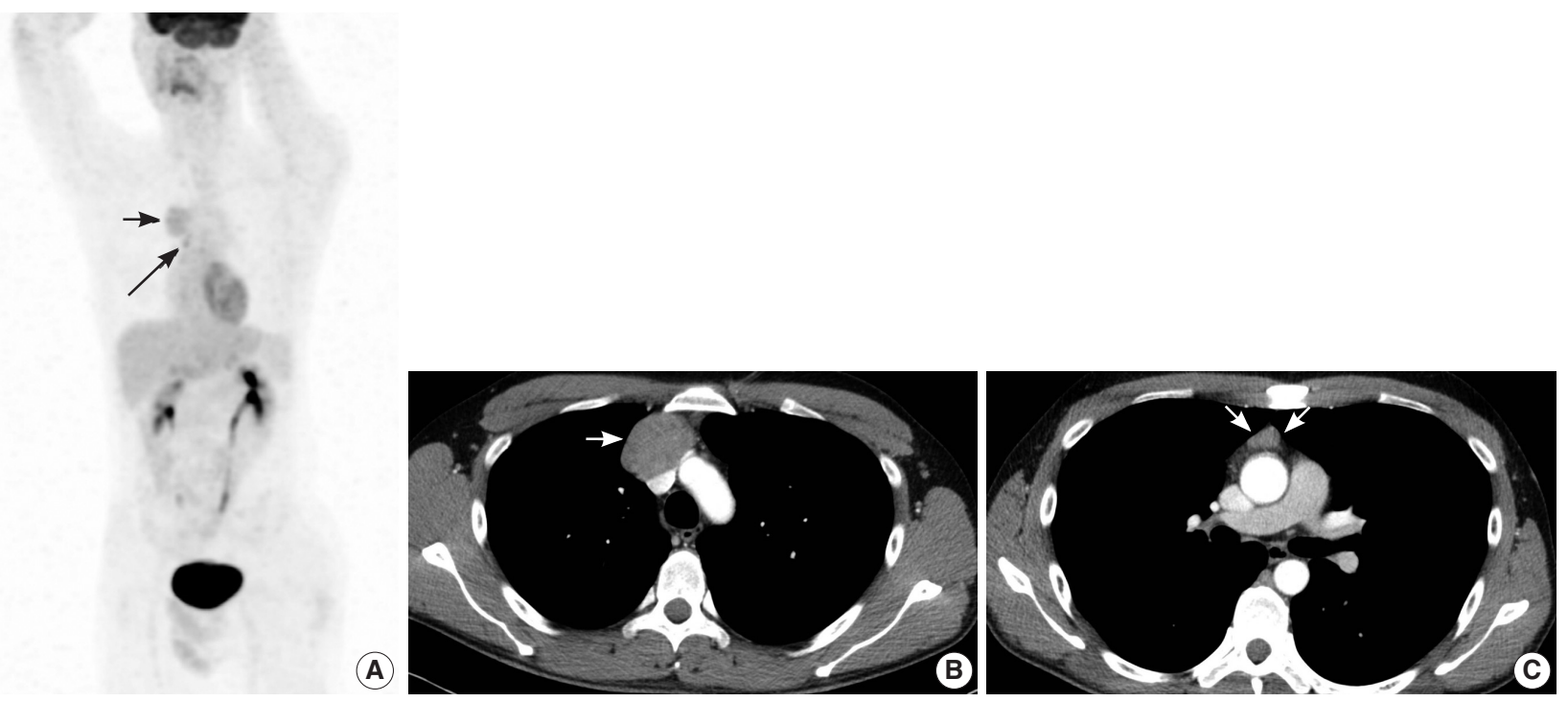

Fig. 1. Radiologic findgings of the anterior mediastinal masses. (A) Maximum intensity projection image of fluorodeoxyglucose-positron emission tomography/computed tomography (PET/CT) shows two faint hypermetabolic foci in the anterior mediastinum. The larger one (short arrow) is $4.8 \mathrm{~cm}$ (standard uptake value [SUV], 3.3) and the smaller one (long arrow) is $2.0 \mathrm{~cm}$ (SUV, 3.4). The smaller one looks like single mass due to limited spatial resolution of PET/CT image (coronal view). (B, C) Axial contrast enhanced chest CT images show multiple tumors in the anterior mediastinum; about 5-cm-sized mass (thymoma, arrow) compressing superior vena cava (B) and $1.5 \mathrm{~cm}$-sized nodule (seminoma, arrows) (C). 


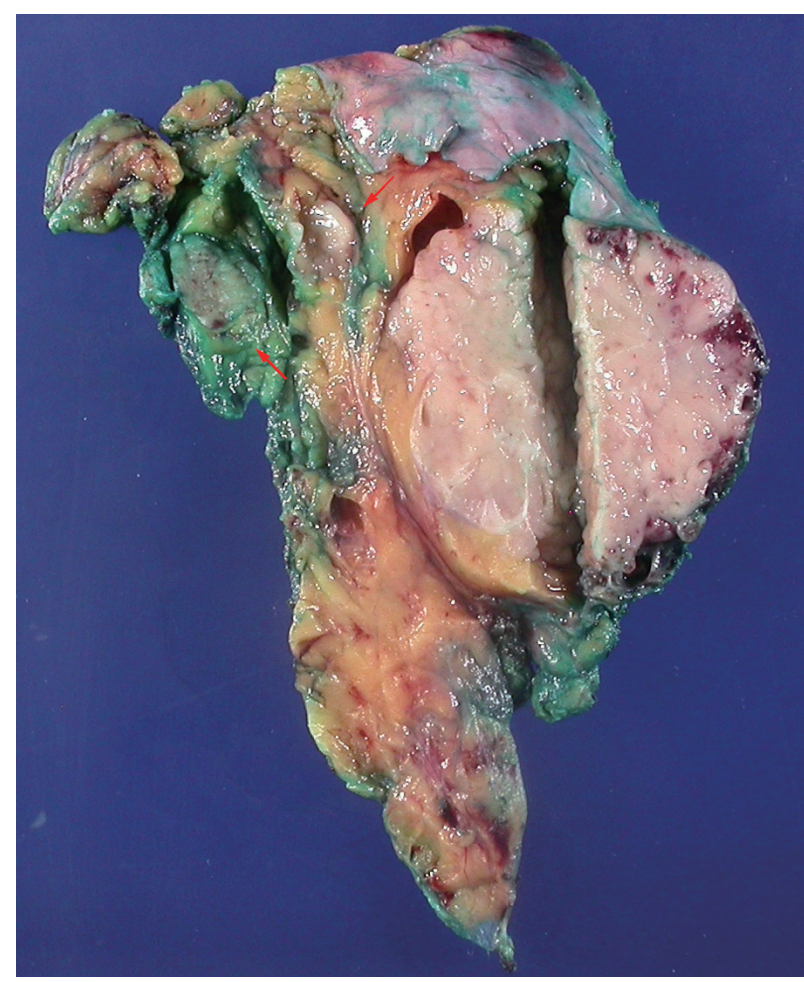

Fig. 2. Macroscopic findings of the thymectomy specimen. Three well circumscribed round masses are seen. The largest one is thymoma and two small round masses (arrows) are seminomas. The seminomas are $1.5 \mathrm{~cm}$ and $0.5 \mathrm{~cm}$ apart from the thymoma.

mass was $0.5 \mathrm{~cm}$ away (Fig. 2). On histologic examination, lowpower magnification of the largest mass showed a well-circumscribed, thick-walled capsule with a partially incomplete area, lobular architecture, and perivascular spaces containing proteinaceous fluid and lymphocytes. The tumor was composed of a dual population of lymphocytes and epithelial cells in equal proportions. Under high-power magnification, the epithelial cells had a bland nuclear appearance with small inconspicuous nucleoli. The lymphocytes that were intermixed with the epithelial cells were small and mature-appearing. Mitotic figures were not identified within either the epithelial cells or the lymphocytes. These histologic patterns are characteristic of a type B2 thymoma (Fig. 3A, B). The two small masses without a capsule revealed clusters or islands of large epithelioid cells with prominent nucleoli and pale cytoplasm that were intermingled with small, infiltrating lymphocytic cells. These findings are similar to the characteristics of seminomas in the testes. Interestingly, these two small tumors revealed a prominent lymphoid follicular hyperplasia with the formation of germinal centers, some microscopic cysts, and Hassall's corpuscles in the surrounding area of the seminomatous component (Fig. 3C, D).

On immunohistochemical staining, the epithelial cells of the thymoma showed diffuse positive reactions for cytokeratin 19 and p63 and negative reactions for placental-like alkaline phosphatase, CD5, CD117, and D2-40 (Fig. 3E, F). The lymphoid cells were positive for TdT. In contrast, the epithelial cells of the seminoma-like tumors showed diffuse positive reactions for CD117, partially strong positive reactions for placental alkaline phosphatase (PLAP) and D2-40, and negative reactions for $\alpha$-fetoprotein, cytokeratin 19, and p63 (Fig. 3G, H). Epithelial membrane antigen staining was observed in Hassall's epithelial cells of the thymoma and in microcystic epithelial cells of the seminomas. In summary, histological and immunohistochemical analyses of the anterior mediastinal masses revealed a type B2 invasive thymoma and two seminomas. The results of the immunohistochemical staining are summarized in Table 1. A biopsy of the testes was not performed.

\section{DISCUSSION}

The simultaneous occurrence of a thymoma and seminoma in the thymus has not yet been reported except for two cases described by Weissferdt et al. ${ }^{8}$ in 2014 . However, they reported a thymoma with a component of a thymic seminoma combined within a single tumor. The seminomatous component showed seminoma-like epithelioid cells with distinct hyalinization adjacent to the area of the conventional thymoma. Our case included one large thymoma and two small seminomas in the anterior mediastinum, with distances of $1.5 \mathrm{~cm}$ and $0.5 \mathrm{~cm}$ between the thymoma and the seminomas. Because multiple thymic masses containing a thymoma and small seminomas are very rare, the possibility of metastasis from another organ or a morphological transformation of the thymic epithelial tumor cells into thymic carcinoma should be considered. The two small masses showed negative reaction for $\mathrm{CD} 5$ and pan-cytokeratin and positive reaction for PLAP and D2-40 by immunohistochemical staining. Although CD5 loss in thymic carcinoma is known, ${ }^{12}$ the other immunohistochemical findings favor seminoma rather than thymic carcinoma. We could not find a case of a thymic carcinoma with a positive reaction for PLAP or D2-40 in the literature. Our case showed site-associated features such as the presence of multiple cysts, reactive remnant thymic epithelium, epithelioid granulomatous reaction, fibrosis, and follicular lymphoid hyperplasia. ${ }^{10,11}$ In the seminomatous tumor, no evidence of metastasis was found in the clinical workup. Fortunately, siteassociated morphologic features of mediastinal masses are rare in gonadal tumors. Although a biopsy of the testes was not performed, metastatic seminoma in the mediastinum from an intra- 

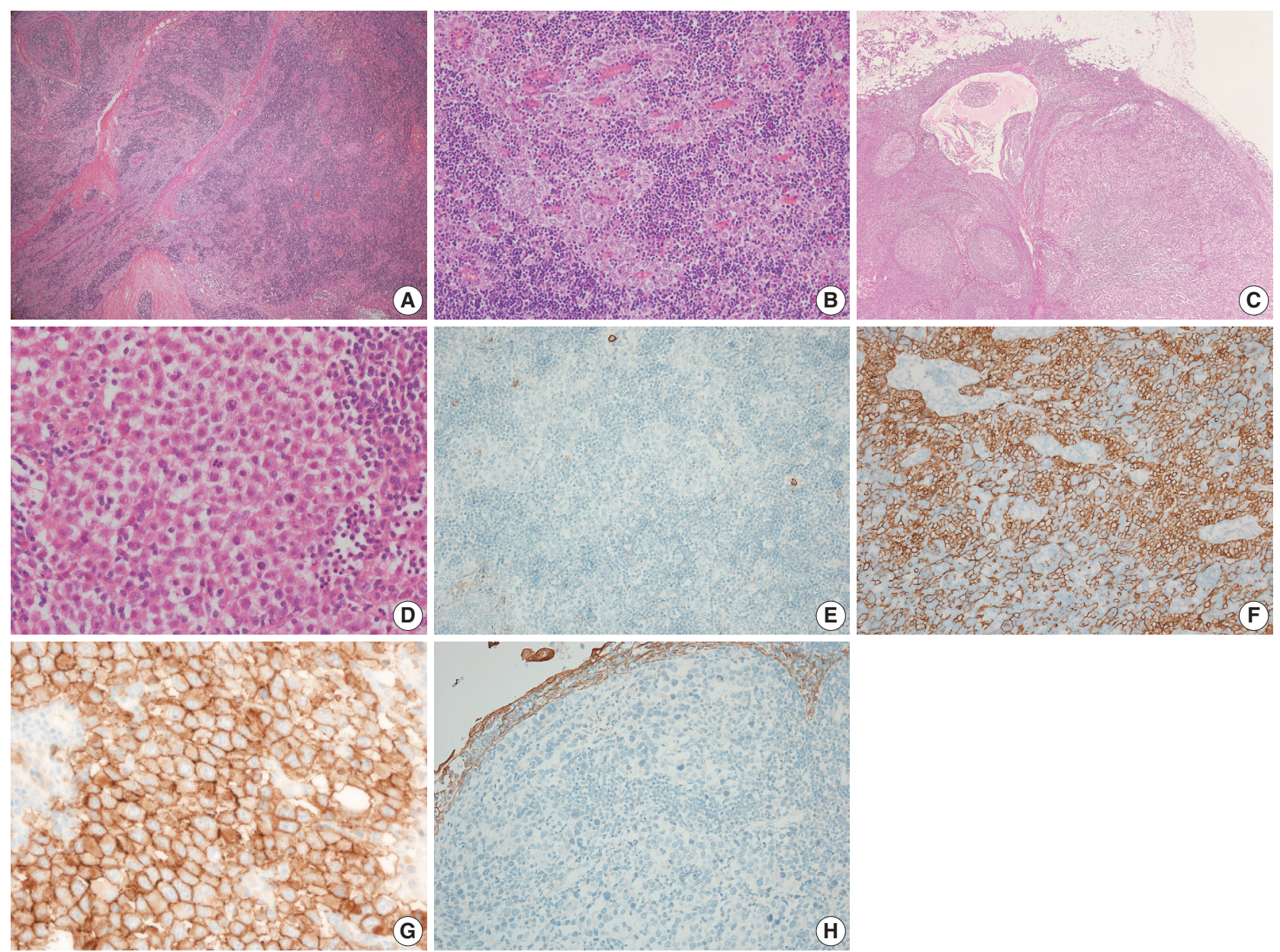

Fig. 3. Histologic findings and immunohistochemical staining results of the thymoma and seminoma of the anterior mediastinal masses. (A) At low power, thymoma shows relatively well demarcated round lobulated mass separated by thick collagenous blands consisting of dual population of lymphocytes and epithelial cells. (B) The epithelial cells show a bland appearance; round to oval with vesicular nuclei, eosinophilic to amphophilic cytoplasm, and small inconspicuous nucleoli. (C) Mediastinal seminoma show numerous lymphoid follicles with germinal center and microcystic change in left area. Seminoma component is seen in right area. (D) Seminoma cells displaying their classical features; indistinct cell border, clear to eosinophilic cytoplasm, vesicular nuclei with eosinophilic nucleoli, and increased mitotic activity. (E) CD117 staining reveals negative reaction for epithelial component of thymoma. (F) Thymic epithelial cells of thymoma highlighted by a cytokeratin 19 immunostain. (G) CD117 staining confirming a diagnosis of seminoma. (H) Cytokeratin 19 staining demonstrates thymic remnant associated with cystic change but is negative for seminoma component around thymic remnant.

tubular germ cell neoplasia is unusual in the absence of retroperitoneal lymph node metastasis. ${ }^{13}$ These clinical and histological findings support the conclusion that these seminomas are of mediastinal origin rather than due to metastasis.

Primary mediastinal seminomas were first described in 1955 by Woolner et al. ${ }^{14}$ and have been well recognized for over 50 years. Their histogenesis is controversial. Normal germ cells in the thymus have yet to be identified. However, it is believed that mediastinal seminomas originate from extragonadal germ cells in the thymic gland due to chromosomal abnormalities. ${ }^{15}$ Chromosome 12p abnormalities, which are specific genetic alterations found in testicular seminomas, are also present in mediastinal seminomas. ${ }^{16}$ However, primary mediastinal seminomas show different genetic findings from those of testicular seminomas. Specifically, Przygodzki et al..$^{17}$ reported a unique pattern of a KIT exon 17 mutations in mediastinal seminomas, which are rare in testicular seminomas. This may imply that the mediastinal seminoma develops through different pathways than that of its gonadal counterpart. Regardless of the tumor location, seminomas show KIT protein expression, which is also seen in thymic carcinomas but not in thymomas.

Histopathological analyses show that thymomas have heterogeneous morphological features such as spindle cells, lymphocytic infiltrate, and rhabdomyomatous components. Additionally, 
Table 1. Results of the immunohistochemical studies

\begin{tabular}{lcc}
\hline $\begin{array}{l}\text { Immunohistochemical } \\
\text { marker }\end{array}$ & $\begin{array}{c}\text { Thymoma } \\
\text { component }\end{array}$ & $\begin{array}{c}\text { Seminoma } \\
\text { component }\end{array}$ \\
\hline CK19 & + & - \\
AFP & - & - \\
TdT & + in lymphocytes & - in lymphocytes \\
PAX-8 & - & - \\
D2-40 & - & + \\
CD5 & - & - \\
CD117 & - & + \\
PLAP & - & + \\
\hline
\end{tabular}

CK19, cytokeratin 19; AFP, $\alpha$-fetoprotein; TdT, terminal deoxynucleotidyl transferase; PLAP, placental alkaline phosphatase.

cytokeratin expression in seminoma tumor cells, which reflects the differential potential or the morphological transformation of the seminoma into another nonseminomatous GCTs, has been reported. ${ }^{16}$ Moreover, a thymic carcinoma or seminoma may occur in combination with a thymoma within a single tumor. Therefore, we cautiously hypothesize that thymoma, thymic carcinoma, and seminoma occur from cells of the same origin, such as embryonic stem cells or primordial germ cells, which have the ability to differentiate into a diverse population of cells. They obtain chromosomal and molecular abnormalities such as chromosome 12p abnormalities and KIT mutations and transform from thymic epithelial tumors into GCTs (seminoma) or from seminomas into thymic epithelial tumors. The etiology and pathogenesis of thymomas and seminomas in the thymus are unknown, and further research is required in this area.

Follicular lymphoid hyperplasia of the thymus can be seen in reactive, autoimmune diseases like myasthenia gravis. Normal thymus and thymic epithelial tumors also exhibit germinal centers. ${ }^{18,19}$ In seminoma, follicular lymphoid hyperplasia may be associated with specific antigens produced by the microenvironment of the seminoma. ${ }^{20}$ Weissferdt and Moran ${ }^{9}$ postulated that the specific distribution of antigen-presenting dendritic cells in mediastinal seminomas is related to the pathogenesis of follicular lymphoid hyperplasia.

In short, we report a case of one thymoma and two mediastinal seminomas that developed simultaneously in the thymus of a young adult. The seminomas revealed florid follicular lymphoid hyperplasia and microcystic changes that do not appear in testicular seminomas. In addition, no clinical or radiological evidence of GCTs was found elsewhere. Therefore, even though multiple masses were present, we considered these tumors to be synchronous primary thymic tumors rather than metastases from an occult tumor of the testis. To our knowledge, this is the first description of a synchronous occurrence of a separate thymoma and mediastinal seminomas within the thymus.

\section{Conflicts of Interest}

No potential conflict of interest relevant to this article was reported.

\section{REFERENCES}

1. Moran CA, Suster S. Primary germ cell tumors of the mediastinum: I. Analysis of 322 cases with special emphasis on teratomatous lesions and a proposal for histopathologic classification and clinical staging. Cancer 1997; 80: 681-90.

2. Strollo DC, Rosado de Christenson ML, Jett JR. Primary mediastinal tumors. Part 1: tumors of the anterior mediastinum. Chest 1997; 112: 511-22.

3. Bokemeyer C, Nichols CR, Droz JP, et al. Extragonadal germ cell tumors of the mediastinum and retroperitoneum: results from an international analysis. J Clin Oncol 2002; 20: 1864-73.

4. Moran CA, Suster S, Przygodzki RM, Koss MN. Primary germ cell tumors of the mediastinum: II. Mediastinal seminomas: a clinicopathologic and immunohistochemical study of 120 cases. Cancer 1997; 80: 691-8.

5. Suster S, Moran CA. Primary thymic epithelial neoplasms showing combined features of thymoma and thymic carcinoma: a clinicopathologic study of 22 cases. Am J Surg Pathol 1996; 20: 1469-80.

6. Moran CA, Suster S, Perino G, Kaneko M, Koss MN. Malignant smooth muscle tumors presenting as mediastinal soft tissue masses: a clinicopathologic study of 10 cases. Cancer 1994; 74: 2251-60.

7. Moran CA, Suster S. Mediastinal seminomas with prominent cystic changes: a clinicopathologic study of 10 cases. Am J Surg Pathol 1995; 19: 1047-53.

8. Weissferdt A, Kalhor N, Moran CA. Combined thymoma-thymic seminoma: report of 2 cases of a heretofore unreported association. Hum Pathol 2014; 45: 2168-72.

9. Weissferdt A, Moran CA. Mediastinal seminoma with florid follicular lymphoid hyperplasia: a clinicopathological and immunohistochemical study of six cases. Virchows Arch 2015; 466: 209-15.

10. Burns BF, McCaughey WT. Unusual thymic seminomas. Arch Pathol Lab Med 1986; 110: 539-41.

11. Schantz A, Sewall W, Castleman B. Mediastinal germinoma: a study of 21 cases with an excellent prognosis. Cancer 1972; 30: 1189-94.

12. Ohue Y, Matsuoka S, Kumeda H, et al. Development of combined thymic carcinoma and thymoma in an extrathymic lesion during long follow-up for recurrent thymoma. Mol Clin Oncol 2016; 4: 
$139-42$.

13. Hailemariam S, Engeler DS, Bannwart F, Amin MB. Primary mediastinal germ cell tumor with intratubular germ cell neoplasia of the testis: further support for germ cell origin of these tumors: a case report. Cancer 1997; 79: 1031-6.

14. Woolner LB, Jamplis RW, Kirklin JW. Seminoma (germinoma) apparently primary in the anterior mediastinum. N Engl J Med 1955; 252: 653-7.

15. Chaganti RS, Rodriguez E, Mathew S. Origin of adult male mediastinal germ-cell tumours. Lancet 1994; 343: 1130-2.

16. Sung MT, Maclennan GT, Lopez-Beltran A, Zhang S, Montironi R, Cheng L. Primary mediastinal seminoma: a comprehensive assessment integrated with histology, immunohistochemistry, and fluo- rescence in situ hybridization for chromosome $12 p$ abnormalities in 23 cases. Am J Surg Pathol 2008; 32: 146-55.

17. Przygodzki RM, Hubbs AE, Zhao FQ, O'Leary TJ. Primary mediastinal seminomas: evidence of single and multiple KIT mutations. Lab Invest 2002; 82: 1369-75.

18. Middleton G. The incidence of follicular structures in the human thymus at autopsy. Aust J Exp Biol Med Sci 1967; 45: 189-99.

19. Vetters JM, Macadam RF. Fine structural evidence for hormone secretion by the human thymus. J Clin Pathol 1973; 26: 194-7.

20. Willis SN, Mallozzi SS, Rodig SJ, et al. The microenvironment of germ cell tumors harbors a prominent antigen-driven humoral response. J Immunol 2009; 182: 3310-7. 